\title{
Improving label space usage for Ethernet Label Switched Paths
}

\author{
Luis F. Caro \\ University of Girona \\ Girona, Spain \\ lfcaro@eia.udg.es
}

\author{
Dimitri Papadimitriou \\ Alcatel-Lucent Bell \\ Antwerp, Belgium \\ Dimitri.Papadimitriou@alcatel-lucent.be
}

\author{
Jose L. Marzo \\ University of Girona \\ Girona, Spain \\ joseluis.marzo@eia.udg.es
}

\begin{abstract}
Ethernet is becoming the dominant aggregation technology for carrier transport networks; however, as it is a LAN technology, native bridged Ethernet does not fulfill all the carrier requirements. One of the schemes proposed by the research community to make Ethernet fulfill carrier requirements is Ethernet VLAN-Label Switching (ELS). ELS allows the creation of label switched data paths using a 12-bit label encoded in the VLAN TAG control information field. Previous label switching technologies such as MPLS use more bits for encoding the label. Hence, they do not suffer from label sparsity issues as ELS might. This paper studies the sparsity issues resulting from the reduced ELS VLAN-label space and proposes the use of the label merging technique to improve label space usage. Experimental results show that label merging considerably improves label space usage.
\end{abstract} ELS

Index Terms-Carrier Ethernet, Label space, label merging,

\section{INTRODUCTION}

Over the last couple of years, there has been an increasing demand for bandwidth combined with exponential growth in the number of clients and network applications that require a carrier class infrastructure. This makes it necessary for carrier networks to improve their bandwidth allocation flexibility and provisioning capability. Ethernet is increasingly attracting network providers. The advantages of Ethernet include its highspeed interfaces that range from $10 \mathrm{Mb} / \mathrm{s}$ to $10 \mathrm{~Gb} / \mathrm{s}(100 \mathrm{~Gb} / \mathrm{s}$ being standardized at IEEE 802.3) together with reduced capital expenditures (CAPEX) which allow it to offer more bandwidth per link.

Hence, Ethernet is progressively taking over legacy technologies in the metro (and even core) network space. However, native bridged Ethernet does not provide all the characteristics of a technology designed for these environments. Indeed, in addition to traffic flow aggregation with low complexity requirements, such positioning requires support of higher flexibility in capacity allocation, higher performance in terms of traffic variation and number of sources (compared to the current Multiple Spanning Tree Protocol (MSTP) as specified in IEEE 802.1s), and higher reliability (compared to the current Rapid Spanning Tree Protocol (RSTP) as specified in IEEE 802.1w).

There are two types of approaches to make Ethernet fulfill carrier requirements: approaches that rely only on improving Ethernet control components and approaches that rely on improving both Ethernet forwarding and control components. Ethernet VLAN-Label Switching (ELS), which belongs to the second type, allows to set up (logical) data paths called Ethernet Label Switched Paths (LSP). In ELS, Ethernet frame processing at the intermediate nodes relies on VLAN-label switching capability. The label is encoded as part of an existing Ethernet MAC frame header field: the S-VLAN 12bit identifier (included in the S-TAG). Moreover, as most routers today support VLAN-based sub-interfaces, interfacing with existing router equipment is straightforward. The label has an interface scope and intermediate nodes are capable of performing label switching (more precisely, they translate the incoming S-VID value into an outgoing S-VID value) but not label stacking. Given the scope of the labels, the number of bits limits the number of LSPs that can traverse a given link $\left(2^{12}\right)$.

Therefore, it is necessary to study the scalability of the ELS architecture against the label value space. Other label switching technologies, such as Multi-Protocol Label Switching (MPLS), use a longer field for encoding the label (20 bits), and stacking; therefore, do not show the behavior (label sparsity) as observed with ELS. The main objective of this paper is to study the scalability of the label space of the ELS technology. For this purpose, the performance of routing schemes in terms of label scalability and the applicability of existing techniques that can be used to overcome or reduce label space limitations are evaluated. The rest of this paper is organized as follows. Carrier Ethernet evolution together with ELS are introduced in Section II. Label merging and previous work on label space are explained in Section III. Section IV shows some simulation results with different topologies and connection requests. Finally conclusions are presented in Section V.

\section{CARrier Class Ethernet Evolution}

Ethernet is increasingly attracting service providers as the inter-connection technology of choice for their metroaggregation infrastructures. It is becoming the data link layer technology of choice for interconnecting IP access, (multiservice) edge and even core routers that are progressively populated with GbE interfaces, thus opening the door to a large spectrum of connectivity services ranging from (Virtual) Private Line to (Virtual) Private LAN services. On the other 
side, when positioned as an aggregation technology (e.g. for metro networks), the Ethernet layer inherits the properties of its design, which is oriented towards facilitating interconnection of various segments by a reduced set of bridges. Indeed, Ethernet technology has been invented and deployed for Local Area Networks (LAN).

\section{A. IEEE 802.1 Limitations}

Ethernet is a connectionless broadcast-access technology that relies on the Spanning Tree Protocol (STP) and its enhanced versions, such as the rapid spanning tree protocol (RSTP), to create and maintain loop free topologies. The bridged Ethernet properties were specifically designed for LAN and other access environments. However, the carrier aggregation networks, where Ethernet is progressively extending, have properties that are not comparable to networks where Ethernet traditionally applies. Such environment, when considering Ethernet as a candidate technology, shall address:

- Ethernet Media Access Control (MAC) address space lookup: Ethernet MAC frame forwarding uses hash-based table lookup that limits MAC table size due to memory consumption and non-deterministic lookup time. Hence, carrier Ethernet frame forwarding shall not make use of any processing that is MAC address dependent but instead be based on MAC address independent switching operation. Moreover, Ethernet aggregation shall ideally be independent of the number of interconnected clients and provide isolation of traffic from different users (with no limitations on the number of clients connected to the network).

- Ethernet MAC address learning: relies on (routing by) flooding of unknown unicast MAC frames, which is appropriate for LAN environments but has several shortcomings when applied in meshed aggregation environments. Firstly, the flooding of unknown MAC frames across the spanning tree topology creates unnecessary processing overhead (aging, filtering, etc.). Secondly, bridges require Filtering Database (FDB) flushing during STP re-convergence, leading to slow recovery.

- Dynamic, flexible and resource-efficient set up of Ethernet data paths. Another major limitation of the current control components for bridged Ethernet networks is its lack of traffic engineering capabilities. Due to the aggregation network size and growth, the number of network links is also increasing. Therefore, the number of blocking links as determined by the spanning tree protocol leads to inefficient use of network resources. PerVLAN spanning tree instance capability (IEEE 802.1s) was added for basic traffic engineering in VLAN bridged Ethernet networks, allowing the use of multiple spanning trees for traffic (belonging to different VLANs) to flow over different paths within the bridged Ethernet network.By using IEEE 802.1s, it is possible to define which VLANs should preferentially use certain links. However, this technique is static and complex to configure (in particular, for meshed environments), and still leads to an inefficient allocation of link resources. In other terms, MSTP usage for traffic engineering purposes is limited in native bridged Ethernet networks. Carrier Ethernet shall provide for route computation and selection (based on various network and service constraints) during provisioning of Ethernet data paths. Using this flexibility, providers can make use of traffic engineering techniques to optimize network resource usage through load sharing and route paths around bottlenecks to less utilized links (i.e. avoid the hyper-aggregation problem).

- Network recovery. The Rapid/Spanning Tree Protocol (IEEE 802.1w/802.1d) being a Distance Vector protocol, has inherent limitations that make "fast recovery" time performance objectives difficult to accomplish. (R)STP is used to construct a loop-free logical tree topology, originated at the root bridge, with leaves and branches spanning all bridges of the entire Ethernet broadcast domain or sub-domain. The IEEE 802.1d STP is based on a break-before-make paradigm. In practice, it takes up to 50 seconds for a bridge to take over from a link failure in an initially blocking port. Subsequent attempts, such as the RSTP, to make it less conservative by considering a make-before-break approach with faster convergence time (in the range of 2 seconds) do not fundamentally solve the initial problem of slow convergence compared to expectations for carrier class network.

In other terms, native bridged Ethernet does not properly address the increasing demand of network providers for scalability (for ensuring wide-scale deployment) and traffic engineering (for ensuring efficient network resource usage and resiliency) required by carrier networks. It should be noted that the Virtual LANs (VLANs), defined in IEEE 802.1Q to subdivide a physical bridged LAN segment into several logical sub-segments by introducing a 12-bit Q-TAG enabling 4096 logical broadcasting sub-domains (and its extension introduced in IEEE 802.1ad, by defining a client 12-bit C-TAG and service 12-bit S-TAG) do not change these observations.

The conclusion is that extending the reach of Ethernet technology toward aggregation networks requires enhancing its properties in order to become a carrier grade technology. Carrier Ethernet, considered in this paper, refers to the technology resulting from the extensions of the native bridged Ethernet forwarding and/or control plane components to address the needs of aggregation networks.

\section{B. Ethernet VLAN-Label Switching (ELS)}

Several solutions have been defined to fulfill carrier Ethernet requirements. For carrier aggregation networks, the Ethernet bridging paradigms have demonstrated several shortcomings (see Section II-A). Henceforth, carrier Ethernet shall depart from both bridged Ethernet forwarding and control components. Two techniques are currently receiving attention from the industry: Provider Backbone Bridge - Traffic Engineering (PBB-TE) and Ethernet VLAN-Label Switching (ELS). Reasons for not considering PBB-TE have been extensively explained in [1]. 
ELS [2], [3] enables the creation of logical data paths established by using constraint-based routing mechanisms provided by a control plane such as Generalized Multi-Protocol Label Switching (GMPLS). The idea behind this approach is to prevent both the forwarding and the control plane from dealing with any Ethernet MAC address so as to maintain independence and transparency in the data plane addressing space. For this purpose, ELS uses the IEEE 802.1ad standard, which separates the VLAN ID space as it enables an Ethernet frame to have two VLAN IDs instead of just one VLAN-ID (as in IEEE 802.1Q). ELS encodes the label in the Service provider VLAN-ID (S-VID) TAG field. An illustration of an IEEE 802.1ad Ethernet frame is presented in Figure 1. These labels, referred to as S-VID labels, are assigned and interpreted locally. The Ethernet S-VID label space has a link local scope and significance, thus providing for 4096 values per interface. Using this label semantic, Ethernet MAC frame switching based on the S-VID label is performed at any device interface that can process this information field.

Thus, ELS enhances the Ethernet MAC frame with the properties of a label switched technology by making its header provide a label semantic. This semantic enhancement of the Ethernet MAC frame header is defined without modifying the IEEE 802.3 frame header format (so as to ensure interoperability with legacy Ethernet switches). The implication is that ELS does not rely on Ethernet MAC address learning (classical Ethernet switches execute this learning process by flooding unknown unicast Ethernet MAC frames) and MAC destination address (DA)-based forwarding.

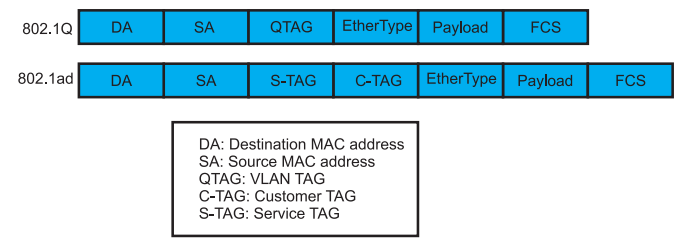

Fig. 1. 802.1ad frame

The logical data paths established using ELS are called Ethernet label switched paths (LSP). Figure 2 describes the label operations along an Ethernet LSP. Intermediate nodes are called Ethernet switching router (E-LSR). The functionality of E-LSRs where the LSP starts and ends is referred to as Ethernet Label Edge router (E-LER). When a native Ethernet frame arrives at the ingress LSR, its E-LER function based on the information of the frame header pushes the correct label (i.e. by adding an S-TAG with the appropriate S-VID value). Then, the Ethernet VLAN-labeled frame is forwarded along the Ethernet LSP. At each E-LSR, the label is swapped (i.e. the incoming S-VID is translated into an outgoing SVID as defined in IEEE 802.1ad). When the frame reaches the egress LSR, its E-LER function pops the label (removing the S-TAG and so the S-VID). Finally, the frame is sent as a native Ethernet frame to its destination. E-LSR are capable of performing swap operations only on labeled frames. The only nodes capable of performing label push and pop are LSRs with LER functionality. The ELS control plane relies on the unified traffic engineering capabilities of GMPLS extended by [2].

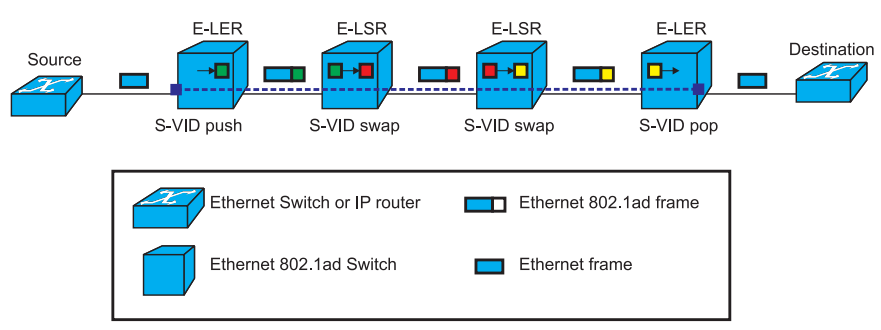

Fig. 2. ELS example

\section{The Label Space Reduction Problem}

Using ELS, labels are encoded using the TCI field of STAGed Ethernet frames. The S-VID value has a length of 12 bits. In comparison, MPLS [4] which is widely deployed in large IP networks, labels are encoded using a 20 bit field per stack level (labels can be stacked in one header) and any node can perform label stack operations. However, E-LSR nodes are not capable of performing stack operations with Ethernet VLAN-labels. In a scenario in which labels have an interface scope, they must be unique per interface. The size of the label space per interface limits the number of LSP that can be forwarded in each link. In ELS, in each link a maximum of $4.096\left(2^{12}\right)$ LSP, can be forwarded. In MPLS, the maximum is $1.048 .576\left(2^{20}\right)$ without considering stacking.

In MPLS, label size has never been considered as a routing limitation like network capacity. With ELS, this aspect must be taken into account. Given that Ethernet VLAN-labels have a significantly smaller size and intermediate nodes, i.e. ELSRs, are not capable of label stacking, it is possible that an LSP request is blocked in certain links even if the unreserved capacity has not reach 0 . Indeed, label space on certain links may have been exhausted before the full capacity of that link has been provisioned. In other words, the label size limitation could represent a new routing constraint, in addition to link capacity. To illustrate this constraint, a comparison between the two technologies is presented in Table I.

\begin{tabular}{|r||l|l|}
\hline & \multicolumn{1}{|c|}{20 bits (MPLS) } & \multicolumn{1}{|c|}{ 12 bits (ELS) } \\
\hline \hline $\mathbf{1 0 M b} / \mathbf{s}$ & $0.0098 \mathrm{~Kb} / \mathrm{s}$ & $2.5 \mathrm{~Kb} / \mathrm{s}$ \\
\hline $\mathbf{1 0 0 M b} / \mathbf{s}$ & $0.098 \mathrm{~Kb} / \mathrm{s}$ & $25 \mathrm{~Kb} / \mathrm{s}$ \\
\hline $\mathbf{1 G b} / \mathbf{s}$ & $0.00098 \mathrm{Mb} / \mathrm{s}$ & $0.25 \mathrm{Mb} / \mathrm{s}$ \\
\hline $\mathbf{1 0 G b} / \mathbf{s}$ & $0.0098 \mathrm{Mb} / \mathrm{s}$ & $2.5 \mathrm{Mb} / \mathrm{s}$ \\
\hline $\mathbf{1 0 0 G b} / \mathbf{s}$ & $0.098 \mathrm{Mb} / \mathrm{s}$ & $25 \mathrm{Mb} / \mathrm{s}$ \\
\hline
\end{tabular}

TABLE I

MPLS AND ELS LABEL GRANULARITY

Table I shows the label granularity values per number of bits used for label encoding (denoted \#bits) and link capacity. The label granularity is calculated by dividing the link capacity by $2^{\# b i t s}$. For reasons of simplicity, the granularities shown in Table I are rounded. For a given technology and link capacity, if there are no connection requests across that link with a bandwidth lower than the label granularity, the probability of a request being rejected due to the sparsity of labels is 0 . In 
a carrier network, with an average link capacity of $10 \mathrm{~Gb} / \mathrm{s}$, it could be said that the acceptable minimum bandwidth for each connection request is equal to or higher than $1 \mathrm{Mb} / \mathrm{s}$ given that traffic is being aggregated. The table entries show that in the case of MPLS the label granularity is lower $(0.0098<1)$ than with ELS $(2.5>1)$. Hence, this example illustrates how the ELS label size could become a routing limitation, while for MPLS it is not.

The label space problem in ELS can be defined as follows: given a routing scenario in ELS, how can the Ethernet LSPs be set up in the network so that corresponding bandwidth requests are not rejected due to sparsity of labels when the unreserved bandwidth in the corresponding links has not reached 0 .

\section{ELS as Intra-domain Aggregation Technology}

A fundamental question remains. Knowing that MPLS has no label constraints, how could ELS technology (based on the same label switching principle but with label size restriction) ever be positioned as an aggregation technology of choice?

The reasons are quite subtle: edge routers are usually interconnected by Ethernet interfaces supporting sub-interfacing (a sub-interface can be configured per C-VID) to aggregation switches, i.e. E-LSRs in the ELS context. Assuming that the IP router-aggregation switch is contained within one administrative domain, the Ethernet MAC address space remains private. The aggregation switch can perform switching based on the Ethernet MAC header of frames exchanged between routers. Nevertheless, the number of interconnected routers can be very high, thus requiring efficient usage of the capacity provided by the aggregation switches and links interconnecting them. The (ingress) edge routers provide for the client flow (first level) aggregation based on the IP destination address and encapsulate the traffic into the tunnel established towards the (egress) edge router. The proposed technique allows for such capability without using MPLS at edge routers and without transporting (inside the aggregation network) the Ethernet traffic over MPLS as defined in [5].

By configuring a C-VID per destination (on the edge router), this technique allows this capability and those provided by MPLS at a reduced cost. Indeed, E-LSRs provide for (second level) traffic flow aggregation without requiring encapsulation of the traffic into Pseudo Wires (PW) over MPLS tunnels. Instead, ELS allows, after upgrading existing Ethernet switches with the capability of processing S-VID per port, to provide for the properties of a carrier environment (if one excludes Operation and Management specifics).

ELS technology is thus promising as an intra-domain aggregation technology when both client devices, e.g. IP routers and E-LSRs, belong to the same administrative domain. The only question that remains is the usability of ELS knowing its label space limitation. The aim of this paper is to investigate how techniques such as label merging help in reducing the ELS label space value sparsity effects.

\section{LABel Merging}

Two techniques can be used in label switching architectures to assign the same label to packets with different sources but the same (intermediate) destination, thus reducing the amount of labels used. These techniques are label merging and label stacking. Label stacking is not discussed further as it cannot by definition be applied to ELS.

Label merging can be used in label switched networks where nodes are capable of performing label swapping. Label merging is able to reduce the number of labels used in a link. It consists of assigning the same label to two or more LSP (that have the same destination) in a continuous and common segment that goes from any common intermediate node to the same destination node. All LSP must follow the same path from the intermediate node to the destination in order to be merged. An illustrative example is presented in Figure 3, where label merging between the two LSP is only possible at link $(5,6)$.

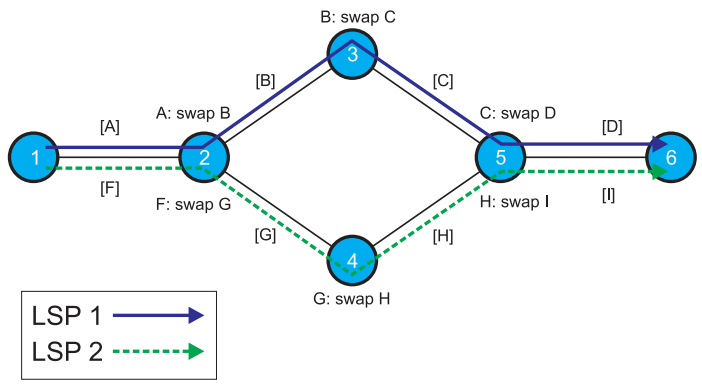

(a) No label merging

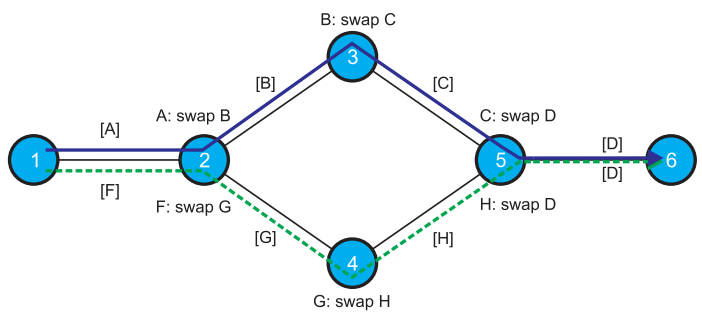

(b) Label merging

Fig. 3. Illustrative Example

To the best of our knowledge, there have not been any label space studies for ELS. Nevertheless, label space reduction has been studied for other architectures like MPLS and All Optical Label Swapping (AOLS). A brief description of these studies is given in this section.

\section{A. Label Space reduction in MPLS}

Label space reduction has been studied in MPLS with the objective of simplifying network management, reducing Operational Expenditure (OPEX) and LSP state message processing.

Two label space reduction scenarios are considered in the literature, with and without re-routing.

The label space reduction with routing scenario is studied in [6]. For a network with $N$ nodes and $M$ edges, [6] presents an offline routing algorithm that uses routing table sizes of at most $(N+M)$ entries, where previous algorithms use at most $(N \times M)$ entries. 
The algorithm receives the network topology, the demand matrix and a given routing solution $S$ as input. Then, the algorithm returns another routing solution $S^{\prime}$ such that for each link $e$ in the network, the load of $e$ in $S^{\prime}$ is no bigger than $S$. If $S^{\prime}$ is optimal to a cost function, $S^{\prime}$ preserves the optimality. $S^{\prime}$ has the characteristic of having table sizes of at most $(N+M)$. The algorithm uses label merging (which means nodes are able to perform label swapping) to reduce the label size. It uses an Integer Linear Program (ILP) based on the multi-commodity flow problem.

Related work that studies the Label Space reduction without routing scenario aims to optimize the number of labels used regardless of the LSP routes (i.e. the LSP routes are already given). In the label space reduction without re-routing scenario the problem is stated as, given a network and a set of LSP routes. The goal is to determine the operations performed at each node (swapping or merging) so that the total number of labels used in the network is minimized.

Previous work by many authors (for instance [7] and [8]) state that the problem of minimizing the label space using label merging in MPLS cannot be solved optimally with a polynomial algorithm (NP-complete). However, in [9] it is shown that it is possible to perform label merging in polynomial time with guarantees that the optimal solution is always found.

\section{B. Label Space reduction in AOLS}

All Optical Label Swapping (AOLS) [10] is an optical packet switching (OPS) architecture. AOLS nodes are capable of forwarding labeled packets without performing opticalelectrical conversions.

The cost of deploying AOLS grows linearly with the number of labels the network is able to support. In AOLS label space reduction represents a top priority over QoS traffic parameters. Unlike in MPLS, in which labels are not a sparse resource, in AOLS routing schemes designed for reducing the number of used labels in the network instead of optimizing TE metrics (such as hopcount) are needed.

Considering this fact [11] studies the label space reduction problem together with the routing problem in AOLS, it also studies the label merging technique. The objective is to reduce the total number of labels used in the network.

In [11] a mathematical model that aims to quantify the number of labels needed for a given set of connection request is also proposed. Simulations comparing the number of labels used with and without label merging show that almost 50\% of the labels can be reduced by using label merging.

\section{Comparison with related work}

Label space reduction has mainly been studied in two different architectures: MPLS and AOLS. In each one, the label space reduction problem has been defined, and each definition has its own objectives and preconditions. The major difference between previous studies and this paper is in the main objective: in both the MPLS and AOLS label space reduction studies, the main goal has been to reduce or minimize the total number of labels; however, in this paper study of ELS the goal is to optimally use a 12 bit label.

\section{Simulation RESUlts AND ANALYSis}

In this section the performance of ELS in the offline and online routing scenarios is evaluated. In the online scenario, given the topology of the network, a set of already established Ethernet LSP and a new incoming bandwidth request between two nodes, the problem is to find a path across the network that satisfies the bandwidth request. The objective is to decrease the probability of future bandwidth requests being blocked. In the offline scenario, given the same topology of the network and a [source-destination] matrix describing the entire network traffic, the problem is to find a set of paths capable of routing all (or part) of the traffic as described by that matrix. The objective is to increase the network's overall throughput. In this scenario, the traffic from a given source-destination can be routed by any number of LSP.

In order to effectively obtain meaningful results three topologies of different sizes are considered: Cost266, Germany50, and Exodus. These topologies are described in terms of number of nodes and number of links in Table II. The table also shows the number of nodes chosen as ingress-egress for the connection requests.

\begin{tabular}{|c|c|c|c|c|}
\hline Name & \# nodes & \# links & \# ing-egr nodes & Source \\
\hline \hline Cost266 (LT) & 37 & 57 & 14 & {$[12]$} \\
\hline germany50 & 50 & 88 & 20 & {$[13]$} \\
\hline Exodus(US) & 79 & 147 & 31 & {$[14]$} \\
\hline
\end{tabular}

TABLE II

TOPOLOGY DESCRIPTIONS

Even though ELS labels have link scope, as some switches do not yet support multiple bridging components, simulations using labels with node scope are also considered.

\section{A. Online scenario}

The existing routing algorithms considered in these simulations are the Shortest Path First (SPF), the Constraint Shortest Path First (CSPF), and the minimum interference routing algorithm (MIRA).

The implemented CSPF selects the path with the minimum TE-Metric. If several paths with the minimum TE-Metric are found, the one with the maximum residual capacity is selected. If there are several with the maximum residual capacity, then the one with the minimum number of hops is selected. The SPF selects the path with the minimum cost metric. If several paths with minimum cost metric are found, the one with the minimum number of hops is selected. The minimum interference routing algorithm (MIRA) is introduced and explained in [15].

For all the topologies the link capacity is set to $10 \mathrm{~Gb} / \mathrm{s}$ and for each topology two sets of bandwidth requests serve as input.

- In the first set, each bandwidth request is set to $1 \mathrm{Mb}$. For this set, the source-destination pairs (ingress-egress node pair) are selected randomly using a uniform distribution. 
The objective of using this set is to obtain a lower bound on the performance of the algorithms.

- In the second set, the bandwidth of each request is selected from the set $1 \mathrm{Mb}, 2 \mathrm{Mb}, 10 \mathrm{Mb}, 20 \mathrm{Mb}$. The source-destination pairs and bandwidth of each request are selected randomly using a uniform distribution.

The algorithms performance is evaluated in terms of the sum of the accommodated bandwidth of all the established LSPs in the network (Throughput), and the number of used labels of the link with the highest amount out of all the links in the network (maximum number of used labels). Each algorithm is evaluated in 5 different scenarios; 1) without any label limit; 2) with 12 bit label limit per link 3) with a 12 bit label limit per link when applying merging 4) with a 12 bit label limit per node 5) with a 12 bit label limit per node when applying merging. The scenario when there is no label limit and label merging is applied is not considered because its throughput is the same from 1).

Results are presented in Table III. When considering homogenous bandwidth requests of $1 \mathrm{Mb}$, results show that with the label size restricted to 12 bits per link (label limit with labels per link column in Table III), all experimented algorithms result in a decrease in throughput that ranges from $32 \%$ (Cost266 with CSPF) to 50\% (Exodus with CSPF). With a restricted label size but label merging enabled, the resulting throughput (merging with labels per link column in Table III) is identical to the one obtained when using an unlimited label size (no limit column in Table III). When the label size is restricted to 12 bits per node (label limit with labels per node column in Table III), the decrease in throughput ranges from $46 \%$ (Cost266 with CSPF) to 69\% (Exodus with CSPF) and with label merging enabled from $12 \%$ (Germany50 with SPF) to 22\% (Exodus with MIRA). The latter observation applies for all the experimented algorithms. This is an interesting result as it shows that label merging overcomes the label size limits for a link scope when the label granularity is of 2.5 $\mathrm{Mb} / \mathrm{s}$ (in this case). This is not the case when the labels have a node scope where there are limitations even with merging.

When considering heterogeneous bandwidth requests, with the label size restricted to 12 bits per link, none of the experimented algorithms show a decrease in throughput higher than $1 \%$. In addition, when label merging is applied, the maximum number of used labels decreases considerably (from 42 to $57 \%$ ). When the label size is restricted to 12 bits per node, the decrease in throughput ranges from 19\% (Germany50 with MIRA) to 29\% (Exodus with CSPF), and with label merging enabled, from $1 \%$ (cost266 with SPF) to $11 \%$ (Exodus with CSPF).

\section{B. Offline scenario}

For the offline routing scenario an integer linear program (ILP) modeling the multicommodity flow problem was evaluated. For more information on the multicommodity flow problem, the reader is referred to [16]. The model has as an objective function to maximize the total accommodated bandwidth expressed by:

$$
\sum_{i, j, c} f(i, j, c) \quad \forall i, j \in N, c \in C \mid i=S_{c}
$$

Where $N$ is the set of nodes, $C$ is the set of commodities for which $S_{c}$ is the source of the commodity $c$, and $f(i, j, c)$ is the flow of commodity $c$ through the edge $(i, j)$. For each solution of the model, the maximum number of used labels per link and maximum number of used labels per node were calculated when using and not using label merging.

\begin{tabular}{|c|c|c|c|c|}
\hline \multirow{2}{*}{ Topology } & \multicolumn{2}{|c|}{ no merging } & \multicolumn{2}{c|}{ merging } \\
\cline { 2 - 5 } & MLL & MLN & MLL & MLN \\
\hline \hline Cost266 & 141 & 134 & 47 & 47 \\
\hline Germany50 & 120 & 114 & 30 & 28 \\
\hline Exodus(US) & 135 & 92 & 24 & 22 \\
\hline
\end{tabular}

MLL=maximum number of used labels per link and $M L N=$ maximum number of used labels per node.

TABLE IV

OFFLINE RESULTS

Results obtained when using the off-line scenario with the topologies described in Table II are presented in Table IV. The highest maximum number of utilized labels was 141 which is very low compared to the 3955 unused labels (around 3.5\%). This result shows that even without merging, for the offline scenario a 4096 label value space is not a limitation.

\section{CONCLusions}

This paper studies the usage of labels for ELS. Due to the sparsity of the ELS label value space, the label merging technique was proposed as a method for improving label space usage.

To prove the effects of a 12 bit label on the online routing problem, several traditional routing algorithms were implemented and tested. In order to evaluate the scalability of ELS label space, scenarios in which the bandwidth granularity associated with labels is high were considered. Demands of the lowest acceptable amount of traffic for an aggregation technology were used to measure the routing limitations of the label space. For the offline routing problem an ILP was used, and the number of labels needed by the optimal solution was analyzed.

Results for the online routing scenario show that when demands of $1 \mathrm{Mb}$ (which is the acceptable minimum) are considered, a 12 bit label can result in performance degradation when no label reduction techniques are used. However, in networks with a link capacity of $10 \mathrm{Gbs}$ with link scope labels, applying label merging allows performance to be maintained in terms of accommodated traffic load. In other terms, the label merging technique significantly reduces the probability of exhausting the label space before the corresponding unreserved (link) capacity drops to 0 . Nevertheless a 12 bit label limit per node can result in performance degradation even when label merging is used. In the offline scenario, results show that even without applying merging a 12 bit label space does not decrease performance. 
This full text paper was peer reviewed at the direction of IEEE Communications Society subject matter experts for publication in the ICC 2008 proceedings.

\begin{tabular}{|c|c|c|c|c|c|c|c|c|c|c|c|}
\hline \multicolumn{12}{|c|}{ 1Mb request list } \\
\hline \multirow{3}{*}{ Topology } & \multirow{3}{*}{ Alg } & \multirow{2}{*}{\multicolumn{2}{|c|}{ no limit }} & \multicolumn{4}{|c|}{ labels per link } & \multicolumn{4}{|c|}{ labels per node } \\
\hline & & & & labc & limit & & sing & labc & limit & & sing \\
\hline & & TH & ML & TH & ML & TH & ML & TH & ML & TH & ML \\
\hline \multirow{3}{*}{ Cost266 } & SPF & 56 & 10240 & 23 & 4096 & $\overline{56}$ & 3456 & 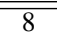 & 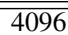 & 38 & 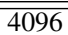 \\
\hline & CSPF & 53 & 10240 & 21 & 4096 & 53 & 3584 & 7 & 4096 & 37 & 4096 \\
\hline & MIRA & 60 & 10240 & 23 & 4096 & 60 & 4096 & 8 & 4096 & 44 & 4096 \\
\hline \multirow{3}{*}{ Germany50 } & SPF & 60 & 10240 & 25 & 4096 & 60 & 4096 & 8 & 4096 & 48 & 4096 \\
\hline & CSPF & 66 & 10240 & 24 & 4096 & 66 & 4096 & 7 & 4096 & 50 & 4096 \\
\hline & MIRA & 60 & 10240 & 24 & 4096 & 60 & 4096 & 9 & 4096 & 48 & 4096 \\
\hline \multirow{3}{*}{ Exodus(US) } & SPF & 76 & 10240 & 35 & 4096 & 76 & 4096 & 10 & 4096 & 61 & 4096 \\
\hline & CSPF & 80 & 10240 & 30 & 4096 & 80 & 4096 & 11 & 4096 & 65 & 4096 \\
\hline & MIRA & 77 & 10240 & 36 & 4096 & 76 & 4096 & 11 & 4096 & 55 & 4096 \\
\hline \multicolumn{12}{|c|}{ Mixed request list } \\
\hline \multirow{3}{*}{ Topology } & \multirow{3}{*}{ Alg } & \multirow{2}{*}{\multicolumn{2}{|c|}{ no limit }} & \multicolumn{4}{|c|}{ labels per link } & \multicolumn{4}{|c|}{ labels per node } \\
\hline & & & & labc & limit & & sing & labc & limit & & jing \\
\hline & & TH & ML & TH & ML & TH & ML & TH & ML & TH & ML \\
\hline \multirow{3}{*}{ Cost266 } & SPF & 90 & 4736 & 89 & 4096 & 90 & 2048 & 70 & 4096 & 89 & 4096 \\
\hline & CSPF & 90 & 4992 & 89 & 4096 & 90 & 2176 & 69 & 4096 & 87 & 4096 \\
\hline & MIRA & 98 & 3968 & 98 & 3968 & 98 & 1792 & 76 & 4096 & 92 & 4096 \\
\hline \multirow{3}{*}{ Germany50 } & SPF & 69 & 3456 & 69 & 3456 & 69 & 1792 & 46 & 4096 & 67 & 4096 \\
\hline & CSPF & 70 & 4480 & 70 & 4096 & 70 & 1920 & 44 & 4096 & 66 & 4096 \\
\hline & MIRA & 68 & 3968 & 68 & 3968 & 68 & 2048 & 49 & 4096 & 61 & 4096 \\
\hline \multirow{3}{*}{$\operatorname{Exodus(US)}$} & SPF & 78 & 6144 & 76 & 4096 & 78 & 2944 & 51 & 4096 & 67 & 4096 \\
\hline & CSPF & 81 & 5632 & 81 & 4096 & 81 & 2560 & 52 & 4096 & 70 & 4096 \\
\hline & MIRA & 78 & 5376 & 78 & 4096 & 78 & 2688 & 52 & 4096 & 71 & 4096 \\
\hline
\end{tabular}

$\mathrm{Alg}=$ algorithm, $\mathrm{TH}=$ throughput $(\%)$ and $\mathrm{ML}=$ maximum number of used labels.

TABLE III

ONLINE RESULTS

Based on the results, we conclude that label merging has to be taken into account in the development of ELS and that novel online routing algorithms that improve label utilization need to be proposed for the case of labels with node scope. This will to be tackled in future research.

\section{ACKNOWLEDGMENTS.}

Part of this work was supported by the Department of Universities, Research and Information Society (DURSI) of the Government of Catalonia, European Social Funds (2005-SGR00296, and, 2007-FIR-00836), and also by the Spanish Science and Technology Ministry (M2R3 - TEC2006-03883) and, the COST project action number 293: Graphs \& Algorithms in Communication Networks.

Part of this work has been carried out within the framework of the TIGER project supported by the Flemish government for Innovation through Science and Technology in Flanders (IWT).

\section{REFERENCES}

[1] D. Papadimitriou. Routing protocols for carrier ethernet networks. In $e$ Photon Workshop, 11th International Conference on Optical Networking Design and Modeling (ONDM), May 2007.

[2] D. Papadimitriou, E. Dotaro, and M. Vigoureux. Ethernet layer 2 label switched paths (lsp). In Proc. of Next Generation Internet Networks, pages 620-621, April 2005.

[3] Lauren Ciavaglia et al. Tiger: Optimizing ip \& ethernet adaptation for the metro ethernet market. In Proc. European Conference in Networks and Optical Communications (NOC 2007), June 2007. Invited Paper.

[4] Eric Rosen, Arun Viswanathan, and Ross Callon. Multiprotocol Label Switching Architecture. IETF, January 2001. RFC 3031.

[5] L. Martini et al. Pseudowire Setup and Maintenance Using the Label Distribution Protocol (LDP). IETF, April 2006. RFC 4447.
[6] D. Applegate and M. Thorup. Load optimal MPLS routing with $\mathrm{N}+\mathrm{M}$ labels. In INFOCOM 2003. Twenty-Second Annual Joint Conference of the IEEE Computer and Communications Societies. IEEE, 2003.

[7] Hiroyuki Saito, Yasuhiro Miyao, and Makiko Yoshida. Traffic engineering using multiple multipoint-to-point LSPs. In INFOCOM (2), pages 894-901, 2000.

[8] S. Bhatnagar, S. Ganguly, and B. Nath. Creating multipoint-to-point LSPs for traffic engineering. High Performance Switching and Routing, 2003, HPSR. Workshop on, pages 201-207, 2003.

[9] Fernando Solano, Ramon Fabregat, and Jose Marzo. On optimal computation of MPLS label binding for multipoint-to-point connections. IEEE Trans. Commun., 2007. Accepted for publication.

[10] Francisco Ramos et al. Ist-lasagne: Towards all-optical label swapping employing optical logic gates and optical flip-flops. Lightwave Technology, Journal of, 23(10):2993-3011, 2005.

[11] Fernando Solano et al. Label merging in all-optical label swapping networks. In Proc. VI Workshop in G/MPLS networks, April 2007.

[12] R. Inkret, A. Kuchar, and B. Mikac. Advanced infrastructure for photonic networks european research project. In Extended Final Report of COST 266 Action, ISBN 953-184-064-4, 2003. p. 21.

[13] S. Orlowski et al. SNDlib 1.0-Survivable Network Design Library. In Proceedings of the Third International Network Optimization Conference (INOC 2007), Spa, Belgium, April 2007. http://sndlib.zib.de.

[14] Neil Spring, Ratul Mahajan, and David Wetherall. Measuring isp topologies with rocketfuel. In SIGCOMM '02: Proceedings of the conference on Applications, technologies, architectures, and protocols for computer communications, 2002. Topologies available at http://www.cs.washington.edu/research/networking/rocketfuel/.

[15] Murali S. Kodialam and T. V. Lakshman. Minimum interference routing with applications to MPLS traffic engineering. In INFOCOM (2), pages 884-893, 2000.

[16] Ravindra K. Ahuja, Thomas L. Magnanti, and James B. Orlin. Network Flows: Theory, Algorithms, and Applications. Prentice Hall, February 1993. 